\title{
Forward mandibular positioning enhances the expression of Ang-1 and Ang-2 in rabbit condylar chondrocytes
}

\author{
ZHAN JING ${ }^{1}$, ZHIYUAN GU ${ }^{2}$ and JIANYING FENG ${ }^{3}$ \\ ${ }^{1}$ Department of Stomatology, Sir Run Run Shaw Hospital, Medical College of Zhejiang University, Hangzhou, \\ Zhejiang 310016; Departments of ${ }^{2}$ Oral and Maxillofacial Surgery and ${ }^{3}$ Orthodontics, School of Stomatology, \\ Zhejiang Chinese Medical University, Hangzhou, Zhenjiang 310053, P.R. China
}

Received March 10, 2013; Accepted July 29, 2013

DOI: $10.3892 / \mathrm{mmr} .2013 .1620$

\begin{abstract}
Functional appliances correct dental malocclusion, partly by exerting an indirect mechanical stimulus on the condylar cartilage, initiating novel bone formation in the condyle. Angiopoietin is involved in the angiogenesis associated with novel bone formation. This study aimed to determine the expression of angiopoietin (Ang)-1 and -2 following forward mandibular positioning (FMP) in the condylar chondrocytes of rabbits. Sixty rabbits (age, 8 weeks) were randomly allocated to the experimental and control groups ( $n=30$ per group). In the experimental group, FMP was induced by a functional appliance. Five rabbits from the experimental group and the control group were sacrificed following 3 days and 1, 2, 4, 8 and 12 weeks, respectively. The right temporomandibular joints (TMJs) were collected and the expression of Ang-1 and -2 was evaluated by immunohistochemical staining. The expression of Ang-1 increased at day 3 and reached a peak at 2 weeks, whereas Ang-2 reached maximal expression 4 weeks after FMP. Subsequently, the expression of Ang-1 and -2 gradually decreased. Thus, FMP enhanced the expression of Ang-1 and Ang-2 in condylar cartilage, which is related to angiogenesis in the process of endochondral ossification.
\end{abstract}

\section{Introduction}

Functional appliances are extensively applied in the treatment of Class II malocclusion by forward mandibular positioning (FMP). A large number of functional appliances were demonstrated to achieve the correction of Class II malocclusion by increasing the mandibular length and rotating the mandibular angle via functional anterior displacement

Correspondence to: Dr Zhan Jing, Department of Stomatology, Sir Run Run Shaw Hospital, Medical College of Zhejiang University, 3 Qingchun East Road, Hangzhou, Zhejiang 310016, P.R. China E-mail: zjuzj1977@163.com

Key words: forward mandibular positioning, functional appliance, endochondral ossification, angiopoietin-1, angiopoietin-2 of the mandible $(1,2)$. FMP provokes cellular and molecular responses in the temporomandiular joint and initiates novel bone formation in the condyle (a growth site) (3). The growth of the mandibular is a process of endochondral ossification in condylar cartilage. During endochondral bone formation, the vascular network in the bone is established by the invasion of capillary sprouts into the newly forming vascular osseous tissue, through a process termed sprouting angiogenesis $(4,5)$. It has been demonstrated that angiopoietin (Ang) is involved in vascularization (6). Autocrine Ang-1/Tie-2 modulates blood vessel plasticity and contributes to vascular maintenance. In addition, Ang-1 enhances survival, migration and network formation of endothelial cells in vitro (7), and induces neovascularization in vivo (8). Ang-2 is a naturally occurring antagonist of Ang-1 that inhibits Ang-1-induced activation of Tie2 (9). Ang-1 and -2 are located at sites of endochondral bone formation in the growing skeleton (10). Moreover, osteoblastspecific expression of Ang-1 in mice leads to an increase in bone mass (11). Since neovascularization is correlated with novel bone formation, it is important to quantitatively assess and compare the pattern of expression of Ang and the quantity of novel bone formation induced by FMP in the condyles with their pattern of expression during natural growth (12). Since the involvement of Ang-1 and -2 during the process of FMP remains to be elucidated, the aim of the present study was to clarify changes in the Ang-1 and -2 expression patterns in condylar chondrocytes during FMP.

\section{Materials and methods}

Experimental animals. A total of 60 Japanese white rabbits (30 male and 30 female; age, 8 weeks; weight, $1.0-1.5 \mathrm{~kg}$ ) were purchased from Zhejiang Experimental Animal Center (Hangzhou, China; Grade II; Certificate SCXK2003-0001). The experiment was in accordance with the Guide for the Care and Use of Laboratory Animals of Zhejiang University (Hangzhou, China) (13). All rabbits were provided with water and a standard diet for 1 week prior to the experiment. The rabbits were randomly assigned to the experimental or control groups ( $n=30$ per group). Food intake and rabbit weight were monitored daily. Five rabbits in the experimental group and five rabbits in the control group were sacrificed at 3 days and 1, 2, 4, 8 and 12 weeks following FMP, respectively. The study 
was approved by the Ethics Committee of Sir Run Run Shaw Hospital, Medical College of Zhejiang University. (Hangzhou, China).

Experimental FMP. The method of experimental FMP was conducted as described previously by Wu et al (13). Briefly, functional appliances, made from polymethylmethacrylate with $20-25^{\circ}$ inclined planes, were cemented to the maxillary central incisors of the experimental group with dental adhesive resin cement [Minnesota Mining and Manufacturing (3M) Co. Monrovia, CA, USA]. The appliances were worn for $24 \mathrm{~h}$ to produce a continuous forward and downward positioning of the mandible. The control group was free from the appliance in order to retain natural growth.

Sample preparation. The right temporomandibular joint (TMJ) samples were fixed in $4 \%$ paraformaldehyde for 3 days and decalcified in $0.5 \mathrm{~mol} / 1$ ethylenediaminetetraacetic acid (EDTA, pH 7.2) for 4 weeks. Subsequently, the TMJ samples were dissected into halves and the connective tissue was removed to expose the areas surrounding the mandibular condyle. Any excess tissue was removed and specimens were embedded in paraffin. Serial sections of 3-5 $\mu \mathrm{m}$ were cut through the TMJ at the parasagittal plane using a rotary microtome (Leica RM 2155, Leica Mikrosysteme Handelsges m.b.H., Vienna, Austria) and hematoxylin and eosin staining was applied. The middle section of the former inclined plane and posterior inclined plane of the condyle was selected.

Immunohistochemistry. Immunohistochemical analysis was performed using the ultrasensitive streptavidin-peroxidase method with a 3'3-diaminobenzidine peroxidase (DAB-PO) kit (Maixin Biotechnology Development Co., Ltd., Fuzhou, China). In brief, for Ang-2 staining, the specimens were treated in $1 \%$ citric acid buffer for $5 \mathrm{~min}$ at a high temperature $\left(120^{\circ} \mathrm{C}\right)$ and pressure $(150 \mathrm{kPa})$, then cooled to room temperature. Subsequent to this, all specimens, including those for Ang-1 and -2 staining,, were treated with $0.3 \%$ hydrogen peroxide to inhibit the activity of endogenous peroxidase. Nonspecific protein staining was blocked by $1.5 \%$ goat serum (Sigma-Aldrich, St. Louis, MO, USA). The tissues were incubated with rat anti-rabbit Ang-1 polyclonal antibody (Santa Cruz Biotechnology Inc., Santa Cruz, CA, USA) and rat anti-rabbit Ang-2 polyclonal antibody (Santa Cruz Biotechnology Inc.) at $4^{\circ} \mathrm{C}$ overnight. The working titer of Ang-1 and -2 was 1:100. Samples were washed with phosphate-buffered saline (PBS) and incubated at room temperature with biotinylated secondary antibody for $10 \mathrm{~min}$. The samples were then washed extensively with PBS, incubated with the streptavidin-peroxidase complex for $10 \mathrm{~min}$ and finally developed according to the manufacturer's instructions (Maixin Biotechnology Development Co., Ltd.). Subsequent to washing in PBS, fresh DAB (Maixin Biotechnology Development Co., Ltd.) was added and the specimens were counterstained with hematoxylin. PBS was used as negative control.

Image and statistical analysis. The immunostained sections were examined using an Olympus microscope (Olympus Co., Tokyo, Japan; magnification, x400) coupled to a video camera (Olympus Co.) and connected to a computer-aided color video image analysis system [High Resolution Pathological Image \& Word Analysis System (HPIAS-1000); Wuhan Qianping Image Technology Co. Ltd., Wuhan, China]. When the images had been captured and digitized onto the video screen, microscopic images were quantitatively analyzed using an image analysis software program (HPIAS-1000; Wuhan Qianping Image Technology Co. Ltd.). Twenty views ( $1 \mathrm{~cm}^{2}$ per view) in a high power field (magnification, $\mathrm{x} 400$ ) were selected randomly. To avoid any biased analysis, the researchers were blinded to the grouping information during the sampling and data analysis. The number of positive cells in the condylar cartilage was calculated and gray values of the expression intensity were measured to indicate the expression of protein. The lower the gray value, the stronger the intensity of the immunostaining. The data were processed with SPSS for Windows (version 16.0, SPSS Inc., Chicago, IL, USA) using the Student's t-test and analysis of variance. Values are presented as the mean $\pm \mathrm{SD}$. $\mathrm{P}<0.05$ was considered to indicate a statistically significant difference.

\section{Results}

Gross examination of animal behavior. The experimental rabbits exhibited difficulties in mastication and consumed less food during the first 2 days after wearing the functional appliance; however, they recovered 2-3 days following surgery. All animals gained weight during the experimental period, and no significant differences in weight were identified between the control and experimental groups.

Expression of Ang-1 and-2 proteins in condylar chondrocytes. Five zones of condylar cartilage (superficial, proliferative, transitive, hypertrophic and mineralized zones) were observed in the control condyle and temporal components. These are marked with $\mathrm{S}, \mathrm{P}, \mathrm{T}, \mathrm{H}$ and $\mathrm{M}$, respectively. In the control condyle, positive signals of Ang-1 and -2 protein expression were observed in the P, T, H and $\mathrm{M}$ zones; however, these were not observed in the superficial zone.

Fig. 1 shows the relative expression of Ang-1 in the experimental and control groups at 12 weeks. A significant decrease of Ang-1 expression (indicated by the significant increase of the mean grey value from 77 to 85 ) was detected only between 0.5 and 1 week. Thereafter, no significant changes were observed between each two adjacent points.. Mandibular advancement resulted in a gradual increase in the expression of Ang-1 when compared with normal growth. Two weeks following FMP, the mean gray value of Ang-1 reached the lowest level of $52.84 \pm 0.32$ in the experimental group, particularly in the $\mathrm{H}$ and $\mathrm{M}$ zones (Fig. 2). Subsequently, the gray value of Ang-1 gradually increased. Compared with the control group, the experimental group exhibited a significantly increased expression of Ang-1 at all time points $(\mathrm{P}<0.05)$.

The relative expression of Ang-2 over time in the control and experimental groups is shown in Fig. 3. In the control group, the mean gray value of Ang-2 was significantly increased following 1 week. However, in the experimental group, a significant decrease in the gray value of Ang-2 was detected from $61.44 \pm 0.21$ to $56.51 \pm 0.57$ at 3 days and 1 week, respectively, following FMP. Ang-2 was continuously upregulated and the gray value reached the lowest level 4 weeks after 


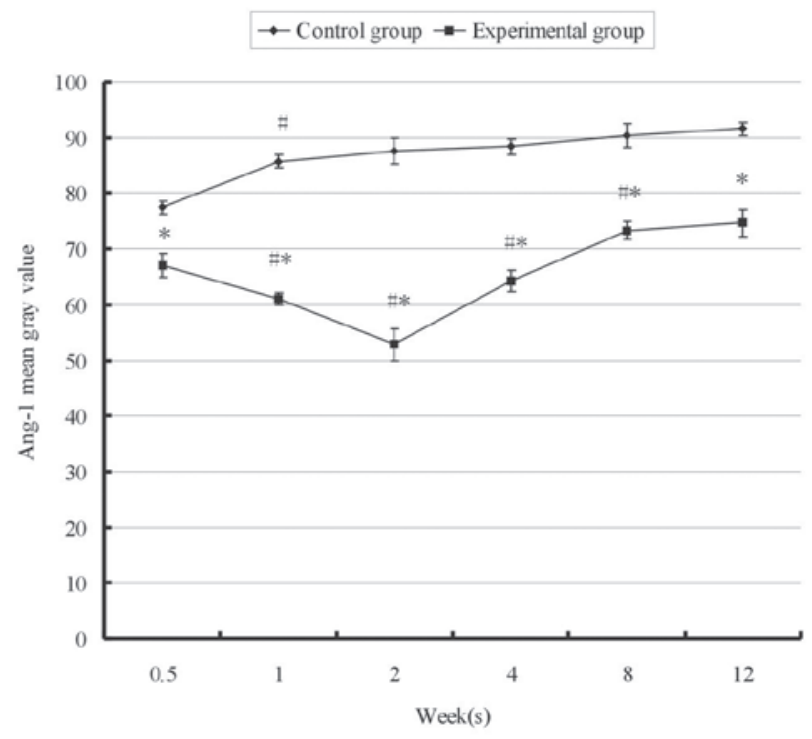

Figure 1. Mean gray value of angiopoietin-1 (Ang-1) vs. time in the cartilage of the control $(n=5)$ and experimental $(n=5)$ groups. Bar represents standard deviation. ${ }^{*} \mathrm{P}<0.05$ compared with the control group and ${ }^{\#} \mathrm{P}<0.05$ compared with the result for the previous time point in the same group.
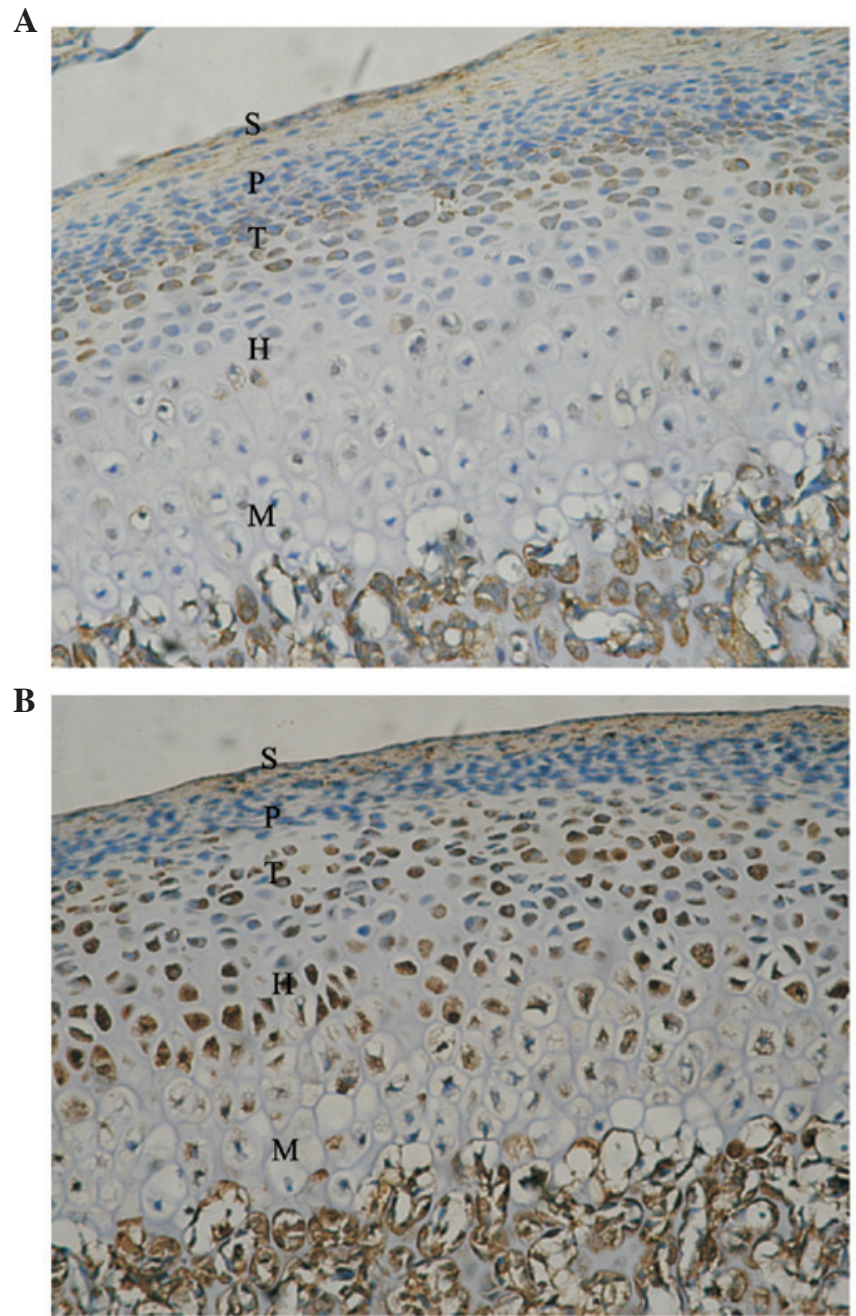

Figure 2. Immunohistochemical micrographs of condylar cartilage in the (A) control and (B) experimental groups 2 weeks following forward mandibular positioning [stain, 3'3-diaminobenzidine (DAB); magnification, x200] Angiopoietin-1-positive cells were identified as tissue with brown staining.

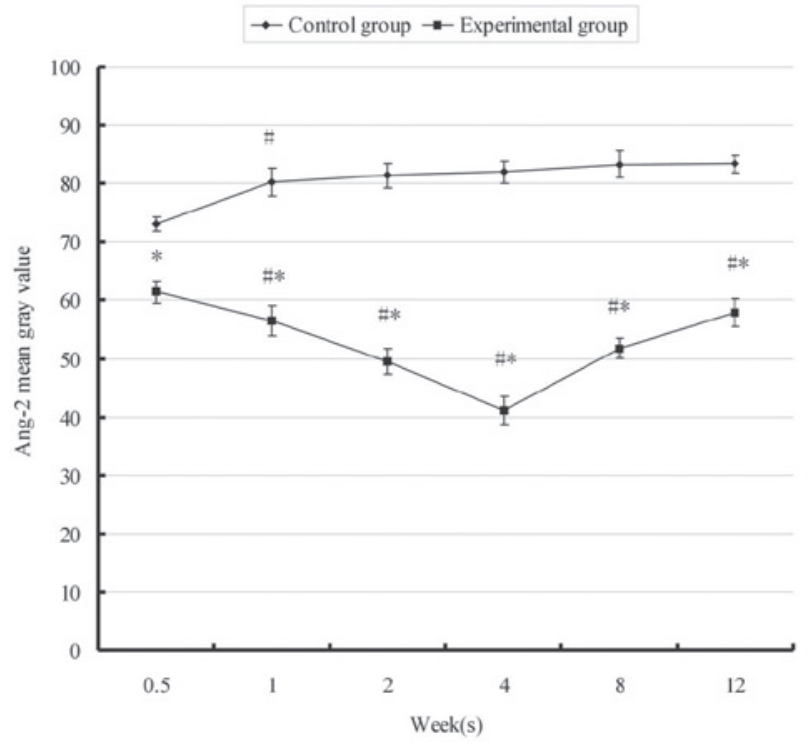

Figure 3. Mean gray value of angiopoietin-2 (Ang-2) vs. time in the cartilage of the control $(n=5)$ and experimental $(n=5)$ groups. Bar represents standard deviation. " $\mathrm{P}<0.05$ compared with the control group and ${ }^{~} \mathrm{P}<0.05$ compared with the result for the previous time point in the same group.
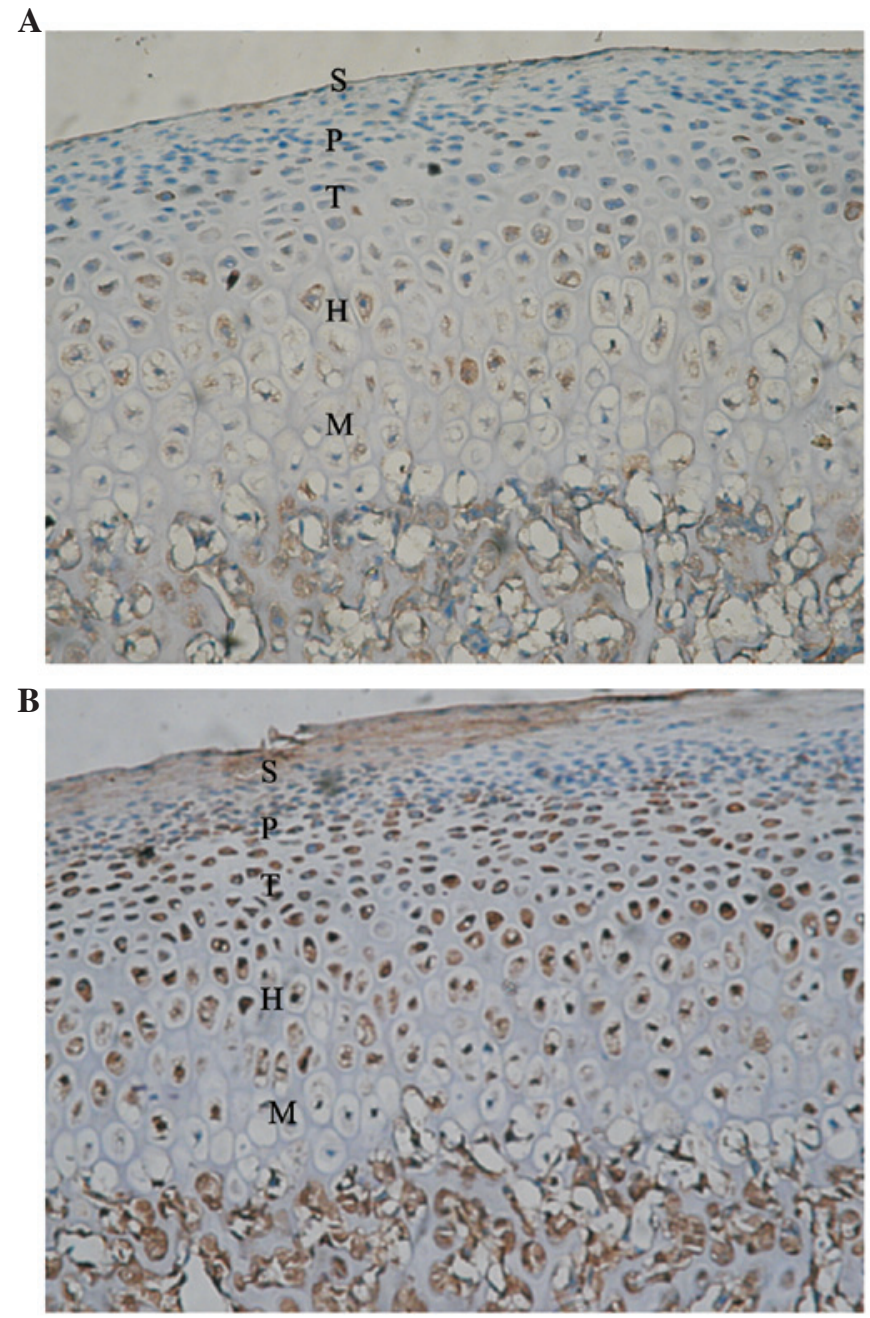

Figure 4. Immunohistochemical micrographs of condylar cartilage in the (A) control and (B) experimental groups 4 weeks following forward mandibular positioning [stain, 3'3-diaminobenzidine (DAB); magnification, $\mathrm{x} 200$ ]. Angiopoietin-2-positive cells were identified as tissue with brown staining. 
FMP, particularly in the M zone (Fig. 4). Statistical analysis demonstrated that the expression of Ang-2 in the experimental group at each time point was stronger than that in the control group $(\mathrm{P}<0.05)$. The expression of Ang-1 and -2 predominantly increased in the posterior region of the condyle.

\section{Discussion}

Ang-1 and -2, members of the angiopoietin family, were demonstrated to be associated with angiogenesis in the present study. The expression of Ang-1 and -2 in the control group marginally fluctuated due to the natural growth of the rabbits. Condyles from the experimental group began to exhibit a progressive increase in the expression of Ang-1 and -2 three days following FMP. In a previous study, Ang-1 was constitutively expressed at low levels in adult vasculature (14). However, pro-angiogenic stimuli, including nitric oxide, hypoxia, tumor necrosis factor and vascular endothelial growth factor (VEGF), led to Ang-1 upregulation (15-17). Ang-2 is a naturally occurring antagonist of Ang-1 and inhibits Ang-1-induced activation of Tie2. An increase in the expression of Ang-2 is an early marker of angiogenesis, as Ang-2 promotes early vascular degeneration and also promotes angiogenesis (18).

The direction of blood supply in the condylar bone plate is radial, pointing towards the mineralized layer of articular cartilage (19). In the present study, the expression of Ang-1 and -2 increased with the maturation of the chondrocytes, particularly in the hypertrophic zone, thus indicating that Ang-1 and -2 may be involved in inducing blood vessel growth into the hypertrophic zone of the condylar cartilage. This expression pattern of Ang-1 and -2 suggests that the invasion of the vasculature is important in bone growth. The expression of Ang- 1 and -2 began to increase from day 3 , and the expression of Ang-1 reached a peak at 2 weeks, while the expression of Ang-2 reached the highest level at 4 weeks. We have previously demonstrated that the peak of bone remodeling during FMP occurred at 8 weeks (20). The expression of Ang-1 and Ang-2 was much earlier than the bone remodeling process. Angiogenesis in bone occurs earlier than endochondral ossification due to the length of the process, which includes vascular invasion, the aggregation and differentiation of mesenchymal cells and endochondral ossification (21). The expression of Ang-2 was stronger and exhibited a longer duration than Ang-1, which may indicate that Ang-2 exhibits a dominant role in the process. Ang-2 was expressed predominantly in the mineralized zone, the site of proliferation for vascular remodeling, and blocked the blood vessel stability effect of Ang-1. Ang-2 has been shown to result in the significant contraction of blood vessels without VEGF; however, with a high concentration of VEGF, the antivascular stabilization effect of Ang-2 promoted novel blood vessel formation $(22,23)$. Rabie et al (24) observed the high expression of VEGF in the mandibular condyle following FMP. The interaction of Ang-2 and VEGF promoted coordination for the formation of blood vessels. A change in Ang-2 expression resulted in vascular instability. This instability in the blood vessels in the presence of VEGF may be involved in promoting angiogenesis, inducing cell differentiation for osteogenesis and promoting the ossification of condylar cartilage and subchondral bone remodeling.
In the present study, in the experimental group, the expression of Ang-1 and -2 predominantly increased in the posterior region of condyle, but not in the anterior and middle regions, which is consistent with the growth direction of novel bone (25). Studies using imaging examination, such as CT and MRI, have demonstrated that the posterior region of condylar growth was markedly greater than the growth in the middle or anterior regions following FMP, and the metabolism in the posterior condyle increased $(26,27)$. Thus, the change of the stress and strain direction in the condyle may have enhanced the expression of Ang-1 and-2 and other related factors, enhanced active osteogenic function and changed the condylar growth direction $(28,29)$. In conclusion, biomechanical stimulation by FMP induced an increase in Ang-1 and -2 expression in condylar chondrocytes. These changes may lead to angiogenesis, which may be involved in the remodeling of the condyle during the treatment of Class II malocclusion.

\section{Acknowledgements}

This study was supported by the National Natural Science Foundation of China (grant no. 81170979).

\section{References}

1. Bishara SE and Ziaja RR: Functional appliances: a review. Am J Orthod Dentofacial Orthop 95: 250-258, 1989.

2. El-Bialy T, El-Shamy I and Graber TM: Growth modification of the rabbit mandible using therapeutic ultrasound: is it possible to enhance functional appliance results? Angle Orthod 73: 631-639, 2003.

3. Ma B, Sampson W, Wilson D, Wiebkin O and Fazzalari N: A histomorphometric study of adaptive responses of cancellous bone in different regions in the sheep mandibular condyle following experimental forward mandibular displacement. Arch Oral Biol 47: 519-527, 2002.

4. Shen G and Darendeliler MA: The adaptive remodeling of condylar cartilage - a transition from chondrogenesis to osteogenesis. J Dent Res 84: 691-699, 2005.

5. Sundaramurthy S and Mao JJ: Modulation of endochondral development of the distal femoral condyle by mechanical loading. J Orthop Res 24: 229-241, 2006.

6. Ribatti D, Vacca A and Presta M: The discovery of angiogenic factors: a historical review. Gen Pharmacol 35: 227-231, 2000.

7. Cascone I, Audero E, Giraudo E, Napione L, Maniero F, Philips MR, Collard JG, Serini G and Bussolino F: Tie-2-dependent activation of RhoA and Racl participates in endothelial cell motility triggered by angiopoietin-1. Blood 102: 2482-2490, 2003.

8. Cho CH, Sung HK, Kim KT, Cheon HG, Oh GT, Hong HJ, Yoo OJ and Koh GY: COMP-angiopoietin-1 promotes wound healing through enhanced angiogenesis, lymphangiogenesis, and blood flow in a diabetic mouse model. Proc Natl Acad Sci USA 103: 4946-4951, 2006.

9. Escobar E, Rodríguez-Reyna TS, Arrieta O and Sotelo J: Angiotensin II, cell proliferation and angiogenesis regulator: biologic and therapeutic implications in cancer. Curr Vasc Pharmacol 2: 385-399, 2004.

10. Horner A, Bord S, Kelsall AW, Coleman N and Compston JE: Tie2 ligands angiopoietin-1 and angiopoietin-2 are coexpressed with vascular endothelial cell growth factor in growing human bone. Bone 28: 65-71, 2001.

11. Suzuki T, Miyamoto T, Fujita N, Ninomiya K, Iwasaki R, Toyama Y and Suda T: Osteoblast-specific Angiopoietin 1 overexpression increases bone mass. Biochem Biophys Res Commun 362: 1019-1025, 2007.

12. Li QF and Rabie AB: A new approach to control condylar growth by regulating angiogenesis. Arch Oral Biol 52: 1009-1017, 2007

13. Wu MJ, Zhan J and Gu ZY: Time course of expression of bcl-2 and bax in rabbit condylar chondrocytes following forward mandibular positioning. Angle Orthod 78: 453-459, 2008. 
14. Fiedler U and Augustin HG: Angiopoietins: a link between angiogenesis and inflammation. Trends Immunol 27: 552-558, 2006.

15. Scott BB, Zaratin PF, Gilmartin AG, Hansbury MJ, Colombo A, Belpasso C, Winkler JD and Jackson JR: TNF-alpha modulates angiopoietin-1 expression in rheumatoid synovial fibroblasts via the NF-kappa B signalling pathway. Biochem Biophys Res Commun 328: 409-414, 2005.

16. Park YS, Kim NH and Jo I: Hypoxia and vascular endothelial growth factor acutely up-regulate angiopoietin-1 and Tie 2 mRNA in bovine retinal pericytes. Microvasc Res 65: 125-131, 2003.

17. Zacharek A, Chen J, Zhang C, Cui X, Roberts C, Jiang H, Teng $\mathrm{H}$ and Chopp M: Nitric oxide regulates Angiopoietin1/Tie2 expression after stroke. Neurosci Lett 404: 28-32, 2006.

18. Oike Y, Yasunaga K and Suda T: Angiopoietin-related/angiopoietin-like proteins regulate angiogenesis. Int J Hematol 80: 21-28, 2004.

19. Aoyama J,Tanaka E, Miyauchi M, Takata T,Hanaoka K, Hattori Y, Sasaki A, Watanabe M and Tanne K: Immunolocalization of vascular endothelial growth factor in rat condylar cartilage during postnatal development. Histochem Cell Biol 122: 35-40, 2004.

20. Zhan J and Gu ZY: Expression of bone histomorphometry parameters in rabbit condyle during mandibular forward positioning. Zhonghua Kou Qiang Yi Xue Za Zhi 48: 303-307, 2013.

21. De Spiegelaere W, Cornillie P, Casteleyn C, Burvenich C and Van den Broeck W: Detection of hypoxia inducible factors and angiogenic growth factors during foetal endochondral and intramembranous ossification. Anat Histol Embryol 39: 376-384, 2010

22. Yee G, Yu Y, Walsh WR, Lindeman R and Poole MD: The immunolocalisation of VEGF in the articular cartilage of sheep mandibular condyles. J Craniomaxillofac Surg 31: 244-251, 2003.
23. Lobov IB, Brooks PC and Lang RA: Angiopoietin-2 displays VEGF-dependent modulation of capillary structure and endothelial cell survival in vivo. Proc Natl Acad Sci USA 99: 11205-11210, 2002.

24. Rabie AB, Leung FY, Chayanupatkul A and Hägg U: The correlation between neovascularization and bone formation in the condyle during forward mandibular positioning. Angle Orthod 72: 431-438, 2002

25. Gu Z, Feng J, Shibata T, Hu J and Zhang Z: Type II collagen and aggrecan mRNA expression by in situ hybridization in rabbit temporomandibular joint posterior attachment following disc displacement. Arch Oral Biol 48: 55-62, 2003.

26. Muto T, Kawakami J, Kanazawa M, Ishii H, Uga S, Yokoyama K and Takeuchi M: Relationship between disc displacement and morphologic features of skeletal Class III malocclusion. Int J Adult Orthodon Orthognath Surg 13: 145-151, 1998.

27. Ishimaru J, Handa Y, Kurita K and Goss AN: The effect of occlusal loss on normal and pathological temporomandibular joints: an animal study. J Craniomaxillofac Surg 22: 95-102, 1994.

28. Huang Q, Opstelten D, Samman $\mathrm{N}$ and Tideman $\mathrm{H}$ : Experimentally induced unilateral tooth loss: histochemical studies of the temporomandibular joint. J Dent Res 81: 209-213, 2002.

29. Hajjar D, Santos MF and Kimura ET: Propulsive appliance stimulates the synthesis of insulin-like growth factors I and II in the mandibular condylar cartilage of young rats. Arch Oral Biol 48: 635-642, 2003 